\section{Pancoast's syndrome due to Pseudomonas aeruginosa infection of the lung apex}

\author{
O Vandenplas, C Mercenier, J-P Trigaux, \\ L Delaunois
}

\section{Abstract}

A case of Pancoast's syndrome was caused by Pseudomonas aeruginosa infection of the lung apex. The infection extended to extrapleural structures of the thoracic inlet.

Pancoast's syndrome includes pain in the shoulder and arm, atrophy of hand muscles, and ipsilateral Horner's syndrome. ${ }^{1}$ It occurs most commonly in patients with a tumour of the lung apex invading the lower brachial plexus roots and the cervicothoracic sympathetic chain. We report a case of Pancoast's syndrome due to Pseudomonas aeruginosa infection of the lung apex with extrapleural extension.

\section{O Vandenplas \\ C Mercenier \\ L Delaunois \\ Department of \\ Radiology \\ J-P Trigaux}

Pulmonary Section

University Hospital of Mont-Godinne, 5180-

Yvoir, Belgium

Reprint requests to:

DrO Vandenplas,

Service de Pneumologie,

Cliniques Universitaires,

Université Catholique de

Louvain, 5180-Yvoir,

Belgium

Accepted 29 March 1991

\section{Case report}

A 67 year old man presented with chronic pain in his left shoulder and upper limb. He was a 40 pack year smoker and had a history of moderate alcohol abuse. He had been adequately treated for pulmonary tuberculosis when he was 25 years old. Twenty two months before this admission, he complained of purulent sputum, left upper chest pain, low grade fever, and weight loss. A $P$ aeruginosa abscess of the left upper lobe was diagnosed and the patient received intravenous antibiotic treatment for three weeks with improvement of his general condition, though chest pain persisted and progressed during the following months to affect the left scapula and the inner aspect of the left arm. Four months before admission he progressively developed pain in the upper interscapular region and paraesthesia in the fourth and fifth fingers of the left hand. Purulent sputum, weight loss, and weakness in the left hand were noted during the month before admission to hospital.

On examination he was afebrile with anhidrosis on the left side of the face and upper chest. An area with increased skin temperature and erythema was noted in the left infraclavicular region of the chest. There was slight miosis of the left pupil and decreased left hand grip without evident muscle wasting. Deep tendon reflexes and pinprick sensation of the upper limbs were normal. No palpable mass was found in the left supraclavicular fossa.

The haemoglobin concentration was $10.3 \mathrm{~g} /$ $\mathrm{dl}$ and the white blood cell count $7 \cdot 1 \times 10^{9} / 1$ with a normal differential. The erythrocyte sedimentation rate was $33 \mathrm{~mm}$ in the first hour. All other blood studies gave results within normal limits. A chest radiograph showed pronounced thickening of the left apical pleura and a thick walled cavity with an air-fluid level in the left upper lobe (fig 1). Computed tomography of the thorax disclosed abnormal contrast enhanced tissue extending from the thickened left apical pleura to the second thoracic vertebral body and infiltrating the posterior chest wall (fig 2). Conventional sagittal tomograms of the spine showed partial collapse of the vertebral body and destruction of the anterior wall of the second thoracic vertebra. Fibreoptic bronchoscopy showed pus originating from
Figure 1 Chest radiograph showing pronounced thickening of the apical pleura and $a$ cavitary lesion with an air-fluid level in the left upper lobe.

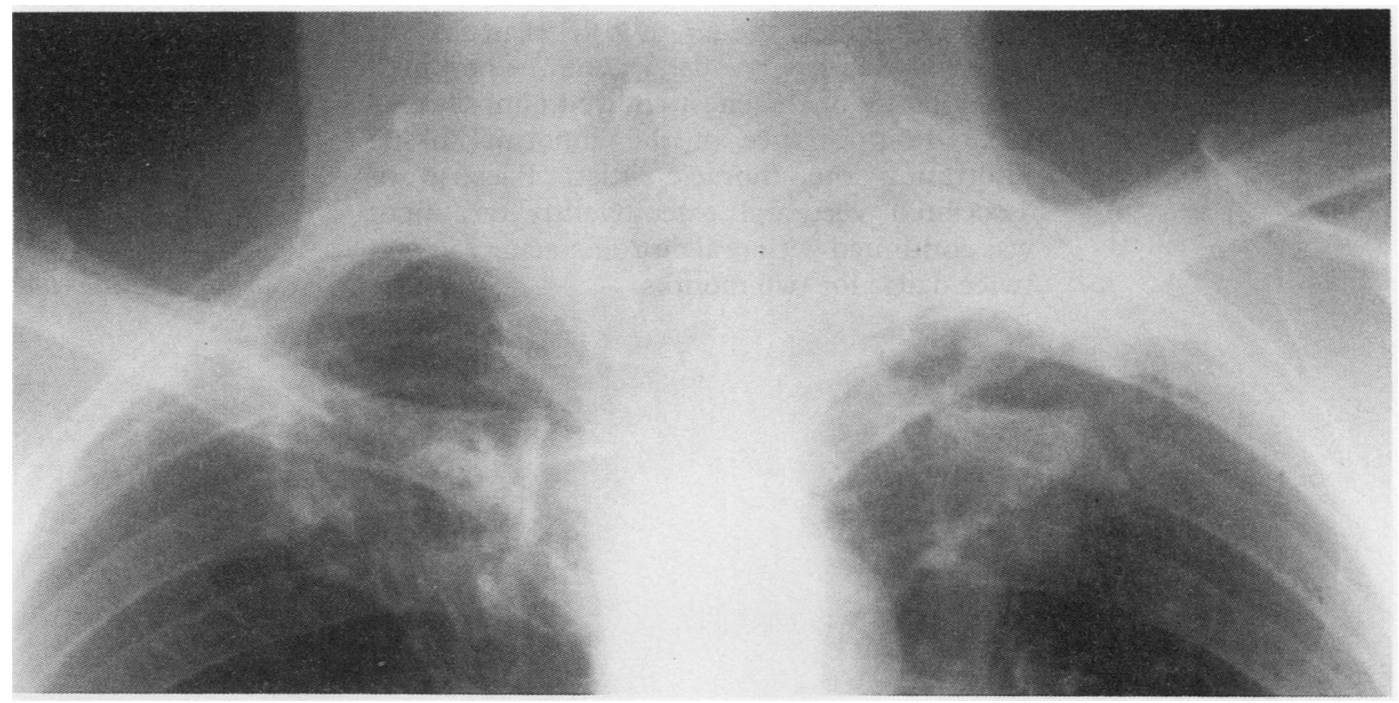




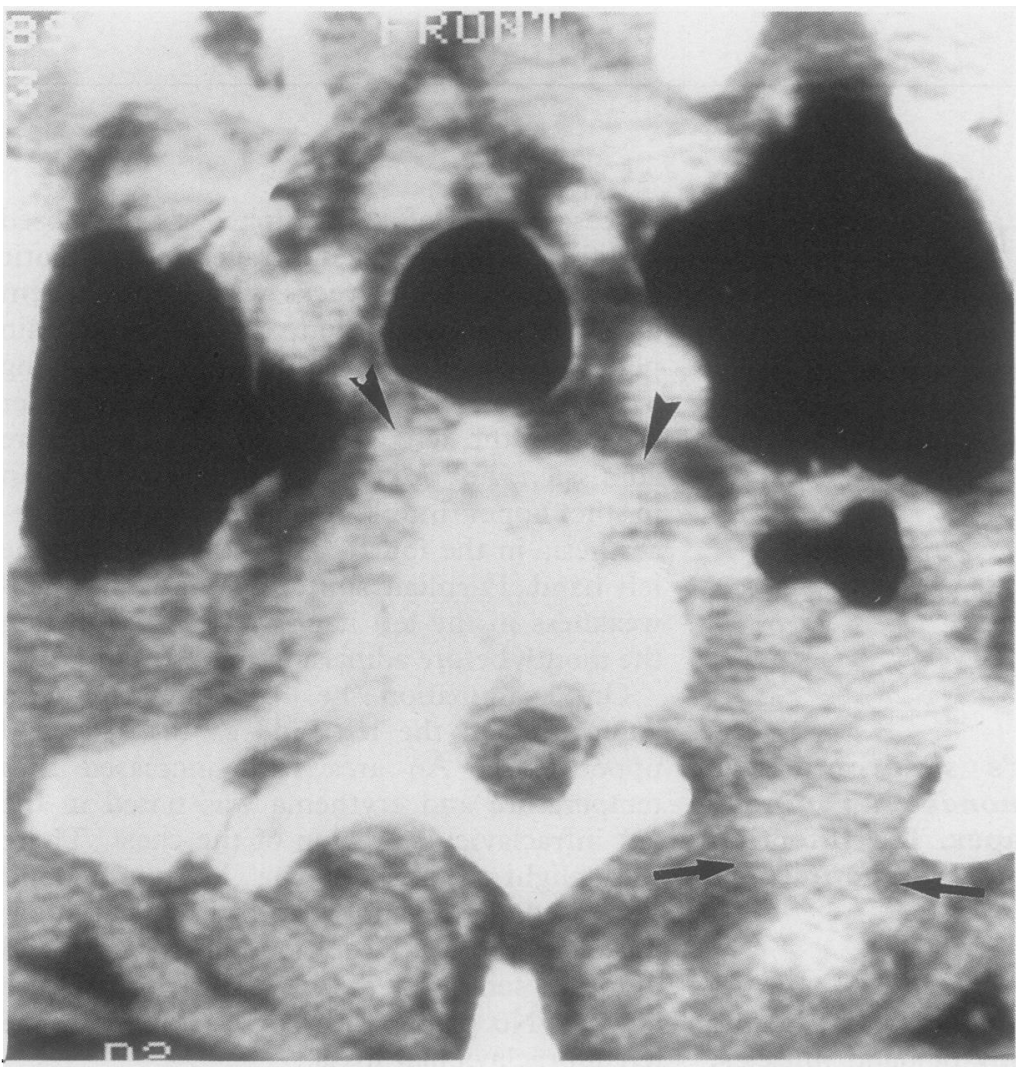

Figure 2 Computed tomogram of the chest: section through the apical regions. Abnormal contrast enhanced tissue is infiltrating the muscles of the posterior chest wall (arrows), the prevertebral soft tissues (arrowheads), and the anterior epidural space; note anterior bone destruction.

\section{Discussion}

Most patients with Pancoast's syndrome have primary lung carcinoma, ${ }^{2}$ though nonpulmonary primary neoplasia and metastatic tumours have been reported. ${ }^{34}$ Benign causes of the syndrome are extremely rare and include pulmonary tuberculosis and actinomycosis, hydatid cysts of the thoracic inlet, ${ }^{6}$ and fungal infections in immunosuppressed patients. ${ }^{7}$

Despite the absence of blepharoptosis and muscle atrophy, there was little doubt that the clinical findings in our patient were similar to those described by Pancoast ${ }^{1}$ and consistent with a lesion of the left brachial plexus roots and the sympathetic chain. Computed tomography suggested that the lesion affecting these nerves was due to abnormal tissue extending from the lung apex. Bacteriological analysis of the percutaneous needle biopsy and bronchial washing specimens indicated that the lesions were caused by $P$ aeruginosa infection. This was confirmed by the clinical response to antibiotic treatment. It is likely that extension of the infectious process to extrapleural structures could have been promoted by the presence of pleural adhesions resulting from previous tuberculosis. The chronic clinical course without fever and without leucocytosis in our patient is not unusual in $P$ aeruginosa infection, particularly in vertebral osteomyelitis. $^{8}$

To our knowledge, this is the first reported case of Pancoast's syndrome due to $P$ aeruginosa infection of the lung apex. It emphasises the need to obtain a precise aetiological diagnosis before treatment decisions are made in patients with Pancoast's syndrome.

lobe Cytological examination of bron washing and brushing specimens were negative for malignant cells. A computed tomography guided percutaneous needle biopsy of the tissue invading the posterior chest wall showed non-specific inflammation and fibrosis. Cultures of sputum, bronchial washing, and percutaneous needle biopsy samples grew $P$ aeruginos $a$ and were negative for mycobacteria. Treatment was started with intravenous ceftazidime $(2 \mathrm{~g})$ and tobramycin $(80 \mathrm{mg})$, both three times a day. Six weeks later all signs and symptoms had resolved except for persistent numbness in the fourth and fifth fingers of the left hand. On a repeat chest radiograph the air-fluid level in the left upper lobe cavity had gone. Computed tomography of the chest showed almost complete disappearance of the abnormal tissue infiltrating the thoracic inlet. Because of associated vertebral osteomyelitis treatment was continued with oral ciprofloxacin $(750 \mathrm{mg}$, twice daily) for two months.
1 Pancoast HK. Superior pulmonary sulcus tumor. JAMA 1932;99:1391-6.

2 Hepper NG, Herskovic T, Witten DM, et al. Thoracic inlet tumors. Ann Intern Med 1966;64:979-89.

3 Brenner B, Carter A, Freidin N, et al. Pancoast's syndrome in multiple myeloma. Acta Haematol 1984;71:353-5.

4 Omenn GS. Pancoast syndrome due to metastatic carcinoma from the uterine cervix. Chest 1971;60:268-70.

5 Stanley SL, Lusk RH. Thoracic actinomycosis presenting as a brachial plexus syndrome. Thorax 1985;40:74-5.

6 Stathatos C, Kontaxis AN, Zafiracopoulos P. Pancoast's syndrome due to a hydatid cyst of the thoracic outlet. $J$ Thorac Cardiovasc Surg 1969;58:764-8.

7 Simpson FG, Morgan M, Cooke NJ. Pancoast's syndrome associated with invasive aspergillosis. Thorax 1986; 41:156-7.

8 Pollack M. Pseudomonas aeruginosa. In: Mandel GL, Douglas RG, Bennett JE, eds. Principles and practice of infectious diseases. New York: Wiley, 1985:1236-47. 